\title{
Lipid, Protein, and MicroRNA Composition Within Mesenchymal Stem Cell-Derived Exosomes
}

\author{
Hao Deng,,"\# Chun Sun, ${ }^{2, \#}$ Yingxin Sun,,\# Huhu Li, Lin Yang, Danbin Wu, Qing Gao, ${ }^{2, \star}$ and Xijuan Jiang ${ }^{2, \star}$
}

\begin{abstract}
Mesenchymal stem cells (MSCs) were regarded as one of the most promising type of seed cells in tissue engineering due to its easy accessibility and multipotent feature of being able to differentiate into adipocyte, osteoblast, cardiomyocytes, and neurons. For years, MSCs have been applied in treating cardiovascular disease, reconstructing kidney injury, and remodeling immune system with remarkable achievements. Basic researches revealed that its clinic effects are not only due to their pluripotent ability but also through their paracrine function that they synthesize and secrete a broad spectrum of growth factors and cytokines. Recent studies show that exosomes is the main paracrine executor of MSCs. The lipid bilayer of exosome maintains its stability and integrity and keeps biological potency of biological substance within it. MSC-derived exosomes were shown to be successful in treating many diseases, including tumor and cardiovascular diseases. However, the exact composition of MSC-derived exosomes is not known yet. In this review, we will discuss the lipid, protein, and microRNA contents within MSC-derived exosomes based on current studies to guide further research and clinical applications of MSC-derived exosomes.
\end{abstract}

Keywords: MSC, exosomes, lipids, proteins, miRNA

\section{Introduction}

$\mathbf{M}$ ESENCHYMAL STEM CELL (MSC) is one of the most widely used stem cells that can be obtained from various tissues such as bone marrow (BM), umbilical cord blood, and fat tissue. MSCs are easily expanded in vitro with immunosuppressive properties (Giordano et al., 2007), so they can be used in allogeneic transplantation. It can differentiate into both mesenchymal cell lineages, including adipogenesis, osteogenesis, and chondrogenesis (Atashi et al., 2015; Coleman et al., 2013), and non-mesenchymal cell lineages such as endothelial, cardiovascular, and neurogenic differentiation (Ikhapoh et al., 2015; Ulrich et al., 2015; Yang et al., 2015). Therefore, MSC was a popular choice for cell therapy of cardiovascular disease, kidney injury, and immune disease. The researches for MSC therapy can be divided into two stages. In the first stage, researchers focus on the transplantation of MSC per se rather than its derived exosomes.

Although the clinic effect is positive, the underlying mechanisms remain obscure. A popular hypothesis is that MSCs would differentiate into the target tissue of the host to repair the damages. However, it was exhibited that the differentiation is insufficient to repair the lesion and most of them died 2 weeks following transplantation (Leiker et al., 2008). Another hypothesis asserts that paracrine function of MSCs is mainly responsible for its therapeutic effect because MSCs synthesize and secrete a broad spectrum of growth factors and cytokines such as vascular endothelial growth factor (VEGF), fibroblast growth factor (FGF), MCP-1, HGF, IGF-I, SDF-1, and so on (Mirotsou et al., 2011).

Furthermore, Gnecchi et al. (2006) exhibited that intramyocardial injection of medium from cultured MSCs can reach the same therapeutic effect in reducing infract size as MSC transplantation. Also, Lai et al. (2011) identified exosome as the active executor in MSC medium as shown by electron microscopy, ultracentrifugation studies, mass spectrometry, and biochemical assays. These lead to the blueprint of the second stage of MSC research: Administration of MSC exosomes instead of MSC into receivers.

Exosomes are lipid bilayer vesicle of endosomal origin with a hydrodynamic radius of $40-100 \mathrm{~nm}$ (Lai et al., 2010). They are produced by numerous cell types like $\mathrm{T}$ cells, dendritic cells, mast cells, tumor cells, and MSCs. Although the functions of exosomes are not well known, they are believed to be significant in intercellular communications. Exosome has been used as an alternative therapeutic agent

\footnotetext{
${ }^{1}$ First Teaching Hospital of Tianjin University of Traditional Chinese Medicine, Tianjin, China.

${ }^{2}$ School of Integrative Medicine, Tianjin University of Traditional Chinese Medicine, Tianjin, China.

${ }^{\#}$ These authors contributed equally to this work and should be considered co-first authors.

*These authors contributed equally to this work and should be considered co-corresponding authors.
} 
for MSC in recent years. Administration of exosomes can avoid many safety concerns caused by MSC transplantation such as arrhythmic (Chang et al., 2006), tumorigenesis, ossification, and/or calcification in tissues (Breitbach et al., 2007).

Besides, the lipid bilayer membrane of exomes is able to protect its contents from degradation by enzymes or chemicals. Therefore, exosomes can maintain the stability, integrity, and biological potency of active substances within them. In this review, we will discuss the specific composition of exosomes derived from MSCs, including lipids, proteins, and microRNAs (miRNAs) (Johnstone, 2006), based on recent researches to provide useful structured information for cell biologists who worked in this field.

\section{Lipids}

\section{Lipids biogenesis in exosomes}

Lipid transport can be mediated by exosomes except carrier proteins (Record et al., 2014). Lipids participate in the multiple steps that determine exosomes' fate and bioactivity. First of all, lipids participate in the biogenesis of exosomes. Intraluminal vesicles, which are the precursors of exosomes, evolved to exosomes through microautophagy with participation of endosomal sorting complex required for transport (ESCRT), heat shock protein Hcs-70, and sorting protein vps4 (Sahu et al., 2011). ESCRT interact with plentiful lipids and enzymes related to lipid metabolism, while vps4 takes part in cholesterol metabolism together with oxysterol binding protein Oshp7 (Wang et al., 2005).

In addition, Hsc-70 specifically binds phosphatidylserine of the endosomal membrane, then ESCRT-III was required to bind the polyglycerol phospholipid bis(monoacylglycero)phosphate (BMP) (Mobius et al., 2003). Finally, BMP participates in exosome production through transphosphatidylation reaction catalyzed by phospholipase D (Record et al., 2011). Besides, cholesterol, oxysterols, ceramides, and lipid transporters have all been shown to be involved in exosome production (Aung et al., 2011; Strauss et al., 2010).

\section{The contents of exosomal lipids}

First of all, exosomes can transport many bioactive lipids as well as lipid metabolism-related enzymes. Exosomes contain a broad spectrum of free fatty acid that is produced either from exosomes itself or laden into exosomes during their biogenesis process (Subra et al., 2010). Monounsaturated and polyunsaturated fatty acid as well as multiple saturated ones are contained in exosomes. Other form of fatty acid, including fatty acid binding proteins (FABP) and lipoproteins, can be taken by exosomes through endocytosis in parental cells. Fatty acid is isolated from MSC-derived exosomes, including leukotrienes, arachidonic acid (AA), phosphatidic acid, prostaglandins lysophosphatidylcholine (LPC), and docosahexaenoic acid (DHA). AA is one of the most abundant unsaturated species. Exosomes also contain enzymes of lipid metabolism, whose accumulation can modulate cell homeostasis of recipient.

For example, three phospholipase A2 (PLA2) and some other enzymes that are relevant to the eicosanoid metabolism were found within MSC-derived exosomes in rat (Fitzner et al., 2011; van der Pol et al., 2016). Notably, all these enzymes in the exosomes are catalytically active. And phospholipase D2 is concerned in exosome release. Com- pared with contents of parental cells, exosomes contain abundant phosphatidic acid (Hullin-Matsuda et al., 2009; Llorente et al., 2013), which are products of phospholipase D or diglyceride kinase activity (Alonso et al., 2011).

\section{Effect of exosomal lipids in target cells}

Exosomes can be part of biomarkers in circulation or can be taken by the proximal or distal cell upon their release. Lipid receptors on the target cells could decide the fate of exosomes. Phosphatidylserine on exosomes can be recognized by target cells through TIM ( $\mathrm{T}$ cell/transmembrane, immunoglobulin, and mucin) receptors, while LPC on exosomes can be recognized by $\mathrm{G}$ protein coupled receptor called G2A. The fate of exosomes depends on the interaction of specific receptors with exosomal PS and LPC phospholipids (Miyanishi et al., 2007). PS on exosomes can bind separately to TIM-1 and TIM-4 receptors to bridge intercellular communication (Freeman et al., 2010).

Exosomal LPC, a chemoattractant for lymphocytes, can attract $\mathrm{T}$ lymphocytes and participate in immune response (Radu et al., 2004), and can induce the maturation of dendritic cells (Perrin-Cocon et al., 2004). Interestingly, when the target cell is undergoing apoptosis, its interaction with exosomal PS and LPS through IgM-type immunoglobulins can lead to exosome elimination (Blanc et al., 2007).

Exosome uptake is mediated by CD36 and CD91, which are scavenger receptor and $\mathrm{Hsp} 90$ receptor, respectively (Record et al., 2014). Once taken by target cell, exosomes can deliver bioactive lipids. Exosomes transport multiple molecules from donor cells into small organelles of recipient cells, including cytosol, nucleus as well as endoplasmic reticulum (ER). Phagocytosis of exosomes can affect the lipid homeostasis and signal pathways of the target cells (Howcroft et al., 2011). Exosome-derived miRNA and mRNA can also affect the translation machinery once taken by the recipient cells (Valadi et al., 2007). It is still a hypothesis that exosomederived lipid molecules were delivered to many cell organelles through soluble carrier proteins, which can be find in cell cytosol and exosome lumen.

AA and DHA in recipient endosomes can be concentrated to 4 and $1 \mathrm{mM}$, respectively, in organelles (Subra et al., 2010). Exosomal AA contributed to the biosynthesis of prostaglandin in ER. On the other hand, exosomal DHA is involved in sterol metabolism in ER. It is reported that DHA derived from exosomes exerts favorable effects to breast carcinoma chemotherapy (Bougnoux et al., 2009). Exosome lumen contains FABP, which can convey DHA from the exosomes to the target cells. When FABP binds AA, it forms FABP-AA complex. Once FABP-AA complex is released from exosomes, it binds to $\operatorname{PPAR} \gamma$ receptor, which was finally targeted to the nucleus where the complex regulates transcriptions of target genes. Although it is widely demonstrated that lipids play a vital role in vesicle stability, the biological and pharmacological effects of lipids derived from MSC exosomes are still to be elucidated.

\section{Proteins}

\section{Common proteins in MSC exosomes}

Mass spectrometry and antibody arrays are the top two methods that detect the proteome of MSC exosomes, the former is limited to large size proteins and the latter is good 
at small proteins like cytokines. Roccaro et al. (2013) screened 32 species of proteins from MSC-derived exosomes of human BM, which were different from BM-MSC-derived cellular proteins. Exosomes may selectively transport proteins to the recipient cells that they interacted with. Exosomes can deliver a significant quantity of proteins to the recipient cells.

Currently, nearly 2000 proteins are identified in MSCderived exosomes from plasma membranes, cytosol, Golgi, and nucleus, and occasionally from the ER or mitochondria (Choi et al., 2013, 2015; Mathivanan et al., 2009). The typical membrane proteins in MSC-derived exosomes include GPI-anchored proteins, tetraspanins such as CD9, CD63, CD82, and CD81, and receptors, for example, tumor necrosis factor receptor 1 . In addition, other proteins capsulated in MSC-derived exosomes include luminal proteins like annexin2, antigen-presentation proteins like MHC-I and MCH-II, cell adhesion molecules like integrin and MFGE8, co-presentation proteins CD86, and cell structure and motility proteins including actin, myosin, and tubulin.

Heat shock proteins and chaperones, including HSP20, HSP60, HSP70, HSP90, and $\alpha \mathrm{B}$-crystalline, metabolism enzymes, including pyruvate kinase, fatty acid synthase, peroxidases, $\beta$-enolase, and glyceraldehyde-3-phosphate dehydrogenase. Proteins related to exosome biogenesis, like ESCRT complex, proteins involved in transcription and protein synthesis such as ubiquitin, histones, transcription factors, and ribosomal protein, and proteins involved in trafficking and membrane fusion proteins like Annexins and Rab protein family. Apart from all these, the proteome of MSC exosomes can be divided into enzymes/ enzyme complexes and signaling molecules.

\section{Enzymes in MSC exosomes}

Today, proteomic studies have shown that MSC-derived exosomes contain five important enzymes involved in glycolysis: glyceraldehyde-3Pdehydrogenase, phosphoglycerate kinase (PGK), phosphoglucomutase, enolase, and pyruvate kinase $\mathrm{m} 2$ isoform (PKm2) (Lai et al., 2011), and several other enzymes such as CD73 (or NT5E or ecto-59-nucleotidase) and 20S proteasome. CD73 is mainly in charge of dephosphorylating extracellular adenosine monophosphate (AMP) into adenosine, which is a crucial activator of Ras/ Raf/mitogen-activated protein kinase and phosphoinositide 3-kinase (PI3K)/protein kinase B (Akt) (Steelman et al., 2011). The component of MSC-derived exosomes can dephosphorylate AMP to adenosine and inorganic phosphate, which provide evidence for the presence of CD73 in the exosomes.

Also, MSC-derived exosomes can phosphorylate the PI3K/Akt signaling pathway and activate adenosine receptors in the existence of AMP (Arslan et al., 2013). In myocardial ischemic/reperfusion (IR) injury model of mice, MSC-derived exosome treatment within 1 hour after reperfusion results in instant and evident increase of cardiac GSK3 and Akt phosphorylation (Lai et al., 2012). All these five glycolytic enzymes from MSC-derived exosomes are responsible for catalyzing five connected reactions of glycolysis, which indicates its potential role in generating glycolytic ATP. When cells are pretreated with oligomycin, which can inhibit mitochondrial ATPase, MSC-derived exosome intervention can increase ATP generation (Zoeger et al.,
2006). Besides, MSC-derived exosomes administrated into myocardial IR model can increase ATP level in the heart tissues (Lai et al., 2011).

\section{Signaling molecules in MSC exosomes}

MSC-derived exosomes contain multiple proteins involved in signaling molecules, like cytokines, interleukins, chemokines, and growth factor. MSC-derived exosomes were shown to be therapeutically efficient in treating myocardial infarction (Lai et al., 2010). This therapeutic efficacy could be mediated by growth factors like VEGF, HGF, FGF, and neuregulin (Formiga et al., 2014; Gu et al., 2010; Palmen et al., 2004) and cytokines as colony-stimulating factors and leukemia inhibitory factor (Okazaki et al., 2007; Zgheib et al., 2012). These factors contribute to reduce fibrosis, tissue revascularization, cardiomyocyte proliferation, and progenitor cell recruitment. Multiple myeloma (MM) patients showed higher levels of IL-6, MCP-1, IGF-1, and $\gamma$-catenin (Gupta et al., 2001; Kumar et al., 2004).

Anderson et al. used HiRIEF LC-MS/MS to investigate the proteomes assembled in human BM stem cell-derived exosomes, and 1927 proteins were identified. Angiogenesisrelated factors, such as platelet-derived growth factor, epidermal growth factor, FGF, and nuclear factor-kappaB signaling pathway proteins, were upregulated in exosomes when MSCs were exposed to ischemic conditions (Anderson et al., 2016). Furthermore, a large amount of cell signaling proteins such as TNF- $\alpha$, TGF- $\beta$, Wnt5, $\beta$-catenin, and delta-like 4 are also known to shuttle between exosomes.

Proteomics of exosomes present a cell line-specific trait. Proteins whose role is basic cellular function, including membrane trafficking, cell junction, cytoskeletal, and chaperone, structural, and so on appeared in all exosomes. By contrast, nucleic acid binding proteins demonstrate great diversity, which might due to the heterogeneity of RNAs between different cell lines. MSC-derived exosomes contained more extracellular matrix proteins than immune cells, which is consistent with the studies that extracellular matrix is a significant regulator of the function and growth of stem cell (Bonnans et al., 2014; Gattazzo et al., 2014).

Therefore, protein composition of exosomes can reflect the physiological and pathological state of its host cell and it can also vary in response to stress and changes in the microenvironment. Salomon et al. found that oxygen concentrations that the MSCs are exposed to can change their exosome protein composition. High oxygen exposure can suppress expressions of proteins related to action cytoskeletal signaling and clathrin-mediate endocytosis (Salomon et al., 2013). Clearly, protein incorporation in exosomes is highly dynamic and might contribute to cellular environment.

\section{microRNAs}

miRNAs are small, noncoding RNA molecules. miRNAs load to exosomes during their biogenesis. Exosomes are rich in miRNAs, with majority in form of pre-miRNAs (Chen et al., 2010). Pre-miRNAs are not active until conversion into mature miRNAs. The secretion of miRNAs from exosomes followed a strictly controlled process dependent on the source and developmental stage of host cells. Multiple miRNAs have been discovered in MSC-derived exosomes. These MSCderived exosomal miRNAs are functional in recipient cells 
(Bang et al., 2014; Halkein et al., 2013; Record et al., 2011; Valadi et al., 2007; Wang et al., 2014).

This revealed that the intercellular communication might be through exchanging exosomal miRNAs between cells. miRNAs encapsulated into exosomes are tightly regulated by various pathophysiological stress stimuli and microenvironment conditions (Jelonek et al., 2016). This allows host cells to produce various kinds of miRNAs in response to different functional requirements and external intervention status. In this regard, certain stress or disease conditions may be reflected by the miRNA content of the exosomes, which can be used to develop biomarkers for the purpose of diagnosis and prognosis. Until now, the function of exosome miRNAs derived from MSCs has been widely studied in researches of tumor, cardiovascular diseases, and so on.

\section{MSC exosomal miRNAs in tumor}

Some researchers found that miRNAs derived from MSC exosomes could promote tumor development. It was demonstrated that exosomes secreted from human MSCs promote breast cancer cell proliferation and metastasis through interacting with miR-21 and miR-3434a, which are protumor miRNAs, as well as about 150 different proteins, most of which are tumor-supportive factors, including plateletderived growth factor receptor- $\beta$ and tissue inhibitor of metalloproteinase (TIMP-1 and TIMP-2) (Vallabhaneni et al., 2015). Another study using human BM-MSC-derived exosomes from normal healthy people and MM patients showed that MM BM-MSCs present a lower content of the tumor suppressor miR-15a and higher levels of oncogene proteins, cytokines, and adhesion molecules compared with exosomes from BM of normal people (Roccaro et al., 2013).

However, debatable conclusions that miRNAs derived from MSC exosomes reduce tumor growth was drawn by Lee et al. (2013) when they found that miR-16, a miRNA known to target VEGF, was enriched in MSC-derived exosomes and is responsible for the antiangiogenic effect in tumor cells. Katakowski et al. (2013) harvest exosomes from miR-146 overexpressing MSCs, and found that its administration can significantly reduce glima xenograft growth in the rat brain tumor model. miR-146 can bind and suppress EGFR mRNA to exert is effect of restraining growth, migration, and invasion of cancer (Katakowski et al., 2010). Although miRNAs were shown enrich in MSC-derived exosomes, their effects on tumor growth varying in different sources of exosomes therefore needed further researches.

\section{Cardioprotection of MSC exosomal miRNAs}

miRNAs in MSC-derived exosomes also function in cardiovascular protection and repair; they have specific roles in inhibiting cardiac apoptosis, regeneration, and fibrosis (Singla, 2016). Furthermore, Wang et al. (2015) identified that miR-223, which is highly enriched in MSC-derived exosomes, plays an important role in cardioprotection using loss-of-function model. Shao et al. (2017) found that the cardioprotective miR-29 and miR-24 were relatively high in MSC-derived exosomes, whereas the cardiac-offensive miR-21 and miR-15 were downregulated in MSC-derived exosomes compared to MSC. miR-29 prevents cardiac fibrosis by reducing the expression of collagen (Wang et al., 2012), while miR-24 relieves aortic vascular inflammation and inhibits cardiomyocytes apoptosis (Maegdefessel et al., 2014; Qian et al., 2011).

Moreover, overexpression of GATA-4 in MSCs significantly increases miR-221 and miR-19a concentration in exosomes, which exert an antiapoptotic effect by inhibiting p53upregulated modulator in cardiomyocytes (Yu et al., 2013). MSCs pretreated with exosomes enriched with miR-22 reduce cardiomyocyte apoptosis following ischemia (Feng et al., 2014). Exosomes derived from MSCs can promote muscle regeneration through enhancing myogenesis and angiogenesis, which is mediated by miR-494 (Nakamura et al., 2015). Another study found that miR-294 enriched in MSC-derived exosomes plays a crucial role in the activation and differentiation of endogenous CSCs in the infracted heart (Khan et al., 2015).

\section{Therapeutic functions of exosomes}

Exosomes can be released by multiple cell types besides MSCs, such as immunocytes, cardiomyocytes, neurons, and cancer cells. Therefore, exosomes can participate in a variety of physiological or pathological process, such as antigen presentation, RNA transportation, tissue repair, neurodegenerative disease development, tumor metastasis, and metabolic reconstruction. Cell-specific proteins, lipids, and nucleic acid in exosomes can act as signaling molecules, which can be transmitted into other cells to modulate their function. Exosomes have become a potential marker for diagnosis and promising treatment choice due to their extensive availability and easy isolation (Naito et al., 2017). As for exosomes derived from MSCs, their therapeutic function is explored in many diseases, most evident in cancer, cardiovascular diseases, and osteoarthritis.

\section{Tumor}

MSCs together with fibroblasts, lymphocytes, inflammatory cells, epithelial cells, and endothelial cells form tumor microenvironment (Quail et al., 2013). MSCs within the tumor microenvironment interact with cancer cells to form the intrinsic communication networks that affect several cancer hallmarks (Hanahan et al., 2011). Recent reports in cancer biology demonstrated that exosomes also play a role as a regulatory agent in such communications. MSCs contribute to the maintenance of cancer dormancy through exosomes (Ono et al., 2014). MSCs transfer miR-23b-containing exosomes into breast cancer cells, then alter their dormant state by targeting myristoylated alanine-rich C-kinase substrate, and modulate cell motility and cell cycle progression (Rombouts et al., 2013).

Consistently, it was reported that MSCs secrete miR-16 through exosomes to downregulate VEGF expression and angiogenesis in breast cancer (Lee et al., 2013). By contrast, several other reports indicate that MSC-derived exosomes can promote tumor growth in renal cancer (Du et al., 2014), gastric cancer, and colorectal cancer (Zhu et al., 2012). Thus, the exact role of MSC-derived exosomes might be cell line dependent. In addition, Roccaro et al. (2013) reported that MSC-derived exosomes of MM patients enhance tumor growth, but MSC-derived exosomes of healthy people suppress tumor growth. In brief, these individual functions of MSC-derived exosomes are dependent on the phenotypes of cancer cells and the donor's physical state. This discovery will provide a novel insight to cancer therapy. 


\section{Myocardial IR injury}

Exosomes secreted by MSCs play essential roles in IR injury (Bei et al., 2017). Study showed that human embryonic stem cell-derived MSC exosomes reduced infarct size followed IR injury in mouse model (Lai et al., 2010). Similarly, it is reported that human MSC-derived exosomes reduced infarct size by $45 \%$ compared to saline treatment. Following IR, exosome treatment increased levels of ATP, $\mathrm{NADH}$, phosphorylated Akt, and phosphorylated GSK-3 $\beta$, while decreased oxidative stress and phosphorylated-c-JNK (Arslan et al., 2013). Also, it is demonstrated that intramyocardial administration of MSC exosomes significantly increases blood flow reperfusion. In line with previous study, it also showed that MSC exosome treatment can lessen infarct size and maintain cardiac systolic and diastolic performance in an acute myocardial infarction of rat model (Bian et al., 2014).

Moreover, Feng et al. (2014) found mouse MSCs can secrete exosomes enriched with miR-22 following ischemic preconditioning (IPC). Exosomes mobilize to cardiomyocytes and prevent further apoptosis due to ischemia. Delivery of IPC exosomes in vivo significantly reduced cardiac fibrosis. It verified a remarkable beneficial role of IPC exosomes in cardiac disease treatment by targeting Mecp2 through miR-22. Yu et al. (2015) revealed that direct intramyocardial administration of exosomes at the border of an ischemic region following ligation of the left anterior descending coronary artery significantly restored cardiac contractile function with reduced infarct size.

Expression of miR-19a was higher in the cardiomyocytes and myocardium treated with GATA-4-overexpressed MSCderived exosomes compared to those treated with control MSC-derived exosomes. The enhanced protective effects were diminished by inhibition of miR-19a. PTEN gene is a predicted target of miR-19. The expression level of PTEN was attenuated in cardiomyocytes treated with GATA-4-overexpressed MSCderived exosomes, which resulted in the activation of Akt and ERK signaling pathways. Wang et al. (2017) revealed that
MSC-derived exosomes conferred excellent cardioprotection. It further certified that exosomal miR-21 can enhance cell survival through the PTEN/Akt pathway.

Mass spectrometry analysis detected all seven $\alpha$ and $\beta$ chains of the $20 \mathrm{~S}$ proteasome, and also the three beta subunits of "immunoproteasome" in a very high confidence level. In a myocardial infarction mouse model, exosomes correlated with a significant drop of oligomerized protein level. Hence, $20 \mathrm{~S}$ proteasome is a candidate exosome protein to ameliorate tissue damage by synergizing with other organelles (Lai et al., 2012). In addition, the beneficial effects of MSC-derived exosomes in reducing myocardial IR injury have also been confirmed by a meta-analysis (Zhang et al., 2016). In summary, MSC-derived exosomes enhance cardiac function and geometry after myocardial IR injury.

\section{Orthopedic diseases}

MSC-derived exosomes have been also studied in orthopedic diseases. After 12 weeks of treatment with $100 \mu \mathrm{g}$ exosomes in osteochondral defect rat model, trochlear grooves of both distal femurs displayed increased gross appearance with raised histological scores. The cartilage and subchondral bone showed characteristic features of complete restoration with including a hyaline cartilage with good surface integrity, complete bonding to adjacent cartilage, and extracellular matrix deposition that closely resemble that of an age-matched unoperated control.

By contrast, only fibrous repair tissues were found in the PBS-treated defects (Zhang et al., 2016). In addition, MSCderived exosomes also show great potential in treating osteoporosis. It is revealed that MSC-derived exosomes increased cell proliferation and alkaline phosphatase activity, and upregulated mRNA transcriptions and protein expressions of osteoblast-related genes in BM-MSCs derived from ovariectomized rats (Qi et al., 2016).

In vivo studies showed that exosomes of human-induced pluripotent stem cell-derived MSCs significantly stimulated bone regeneration and angiogenesis in critical-sized calvarial

FIG. 1. A brief introduction of encapsulated lipids, proteins, and miRNAs produced and released by MSC-derived exosomes ( 40-100 nm). miRNA, microRNA; MSC, mesenchymal stem cell.

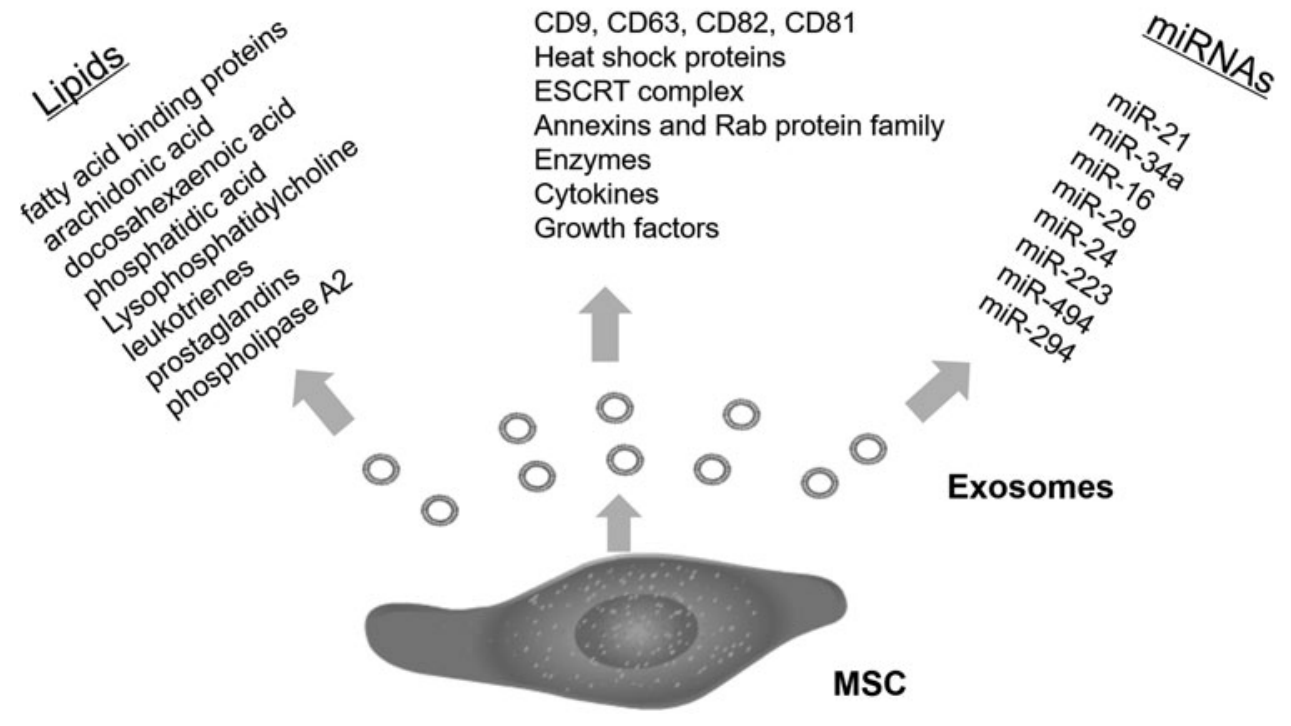


defects in ovariectomized rats. Qin et al. (2016) isolated BMMSC-derived exosomes and tested the influence of the exosomes on osteogenesis both in vivo and in vitro. The results demonstrated that exosomes positively regulated osteogenic genes and osteoblastic differentiation, but did not inhibit cell proliferation in vitro. Also, miR-196a plays an essential role in the regulation of osteoblastic differentiation and the expression of osteogenic genes.

\section{Conclusion}

Together, the study of MSC-derived exosomes is still in the initial stage. Although numerous lipids, proteins, and miRNAs were discovered in MSC-derived exosomes (Fig. 1), there is not a comprehensive summary to understand the complete components of MSC-derived exosomes. The ingredients within MSC-derived exosomes are also dynamic according to surrounding microenvironment and metabolic status of their host cells. Even so, the diagnostic, therapeutic, and other functional characteristics of MSC-derived exosomes will play an important role in biological sciences, bioengineering, as well as in preventing and treating many diseases, including cardiovascular diseases and cancer.

Unfortunately, there is still a huge gap between current research and clinical applications due to the fact that the contents of exosomes are far from being thoroughly characterized. Paradoxically, exosomes may have the potential to advance progress of the disease or accelerate tumorigenesis. Therefore, further studies of understanding how exosomal lipids, proteins, and miRNAs contribute to the biological activity and how we get therapeutic MSC-derived exosomes still need to be elucidated to future application of exosomes in tissue repair.

\section{Acknowledgment}

This work was supported by the National Natural Science Foundation of China (81503504, 81573733, and 81704056).

\section{Author Disclosure Statement}

The authors do not have any conflict of interests to be declared regarding the content of the article. In addition, they do not have direct financial relationship with any commercial identity mentioned in this article.

\section{References}

Alonso, R., Mazzeo, C., Rodriguez, M.C., Marsh, M., FraileRamos, A., Calvo, V., Avila-Flores, A., Merida, I., and Izquierdo, M. (2011). Diacylglycerol kinase alpha regulates the formation and polarisation of mature multivesicular bodies involved in the secretion of Fas ligand-containing exosomes in T lymphocytes. Cell Death Differ. 18, 1161-1173.

Anderson, J.D., Johansson, H.J., Graham, C.S., Vesterlund, M., Pham, M.T., Bramlett, C.S., Montgomery, E.N., Mellema, M.S., Bardini, R.L., Contreras, Z., Hoon, M., Bauer, G., Fink, K.D., Fury, B., Hendrix, K.J., Chedin, F., El-Andaloussi, S., Hwang, B., Mulligan, M.S., Lehtio, J., and Nolta, J.A. (2016). Comprehensive proteomic analysis of mesenchymal stem cell exosomes reveals modulation of angiogenesis via nuclear factor-kappaB signaling. Stem Cells 34, 601-613.

Arslan, F., Lai, R.C., Smeets, M.B., Akeroyd, L., Choo, A., Aguor, E.N., Timmers, L., van Rijen, H.V., Doevendans, P.A., Pasterkamp, G., Lim, S.K., and de Kleijn, D.P. (2013).
Mesenchymal stem cell-derived exosomes increase ATP levels, decrease oxidative stress and activate PI3K/Akt pathway to enhance myocardial viability and prevent adverse remodeling after myocardial ischemia/reperfusion injury. Stem Cell Res. 10, 301-312.

Atashi, F., Modarressi, A., and Pepper, M.S. (2015). The role of reactive oxygen species in mesenchymal stem cell adipogenic and osteogenic differentiation: A review. Stem Cells Dev. 24, 1150-1163.

Aung, T., Chapuy, B., Vogel, D., Wenzel, D., Oppermann, M., Lahmann, M., Weinhage, T., Menck, K., Hupfeld, T., Koch, R., Trumper, L., and Wulf, G.G. (2011). Exosomal evasion of humoral immunotherapy in aggressive B cell lymphoma modulated by ATP-binding cassette transporter A3. Proc. Natl. Acad. Sci. U. S. A. 108, 15336-15341.

Bang, C., Batkai, S., Dangwal, S., Gupta, S.K., Foinquinos, A., Holzmann, A., Just, A., Remke, J., Zimmer, K., Zeug, A., Ponimaskin, E., Schmiedl, A., Yin, X., Mayr, M., Halder, R., Fischer, A., Engelhardt, S., Wei, Y., Schober, A., Fiedler, J., and Thum, T. (2014). Cardiac fibroblast-derived microRNA passenger strand-enriched exosomes mediate cardiomyocyte hypertrophy. J. Clin. Invest. 124, 2136-2146.

Bei, Y., Das, S., Rodosthenous, R.S., Holvoet, P., Vanhaverbeke, M., Monteiro, M.C., Monteiro, V.V.S., Radosinska, J., Bartekova, M., Jansen, F., Li, Q., Rajasingh, J., and Xiao, J. (2017). Extracellular vesicles in cardiovascular theranostics. Theranostics 7, 4168-4182.

Bian, S., Zhang, L., Duan, L., Wang, X., Min, Y., and Yu, H. (2014). Extracellular vesicles derived from human bone marrow mesenchymal stem cells promote angiogenesis in a rat myocardial infarction model. J. Mol. Med. (Berl) 92, 387-397.

Blanc, L., Barres, C., Bette-Bobillo, P., and Vidal, M. (2007). Reticulocyte-secreted exosomes bind natural IgM antibodies: Involvement of a ROS-activatable endosomal phospholipase iPLA2. Blood 110, 3407-3416.

Bonnans, C., Chou, J., and Werb, Z. (2014). Remodelling the extracellular matrix in development and disease. Nat. Rev. Mol. Cell Biol. 15, 786-801.

Bougnoux, P., Hajjaji, N., Ferrasson, M.N., Giraudeau, B., Couet, C., and Le Floch, O. (2009). Improving outcome of chemotherapy of metastatic breast cancer by docosahexaenoic acid: A phase II trial. Br. J. Cancer 101, 1978-1985.

Breitbach, M., Bostani, T., Roell, W., Xia, Y., Dewald, O., Nygren, J.M., Fries, J.W., Tiemann, K., Bohlen, H., Hescheler, J., Welz, A., Bloch, W., Jacobsen, S.E., and Fleischmann, B.K. (2007). Potential risks of bone marrow cell transplantation into infarcted hearts. Blood 110, 1362-1369.

Chang, M.G., Tung, L., Sekar, R.B., Chang, C.Y., Cysyk, J., Dong, P., Marban, E., and Abraham, M.R. (2006). Proarrhythmic potential of mesenchymal stem cell transplantation revealed in an in vitro coculture model. Circulation 113, 1832-1841.

Chen, T.S., Lai, R.C., Lee, M.M., Choo, A.B., Lee, C.N., and Lim, S.K. (2010). Mesenchymal stem cell secretes microparticles enriched in pre-microRNAs. Nucleic Acids Res. 38, 215-224.

Choi, D.S., Kim, D.K., Kim, Y.K., and Gho, Y.S. (2013). Proteomics, transcriptomics and lipidomics of exosomes and ectosomes. Proteomics 13, 1554-1571.

Choi, D.S., Kim, D.K., Kim, Y.K., and Gho, Y.S. (2015). Proteomics of extracellular vesicles: Exosomes and ectosomes. Mass Spectrom. Rev. 34, 474-490.

Coleman, C.M., Vaughan, E.E., Browe, D.C., Mooney, E., Howard, L., and Barry, F. (2013). Growth differentiation 
factor-5 enhances in vitro mesenchymal stromal cell chondrogenesis and hypertrophy. Stem Cells Dev. 22, 1968-1976.

Du, T., Ju, G., Wu, S., Cheng, Z., Cheng, J., Zou, X., Zhang, G., Miao, S., Liu, G., and Zhu, Y. (2014). Microvesicles derived from human Wharton's jelly mesenchymal stem cells promote human renal cancer cell growth and aggressiveness through induction of hepatocyte growth factor. PLoS One 9, e96836.

Feng, Y., Huang, W., Wani, M., Yu, X., and Ashraf, M. (2014). Ischemic preconditioning potentiates the protective effect of stem cells through secretion of exosomes by targeting Mecp2 via miR-22. PLoS One 9, e88685.

Fitzner, D., Schnaars, M., van Rossum, D., Krishnamoorthy, G., Dibaj, P., Bakhti, M., Regen, T., Hanisch, U.K., and Simons, M. (2011). Selective transfer of exosomes from oligodendrocytes to microglia by macropinocytosis. J. Cell Sci. 124, 447-458.

Formiga, F.R., Pelacho, B., Garbayo, E., Imbuluzqueta, I., DiazHerraez, P., Abizanda, G., Gavira, J.J., Simon-Yarza, T., Albiasu, E., Tamayo, E., Prosper, F., and Blanco-Prieto, M.J. (2014). Controlled delivery of fibroblast growth factor-1 and neuregulin-1 from biodegradable microparticles promotes cardiac repair in a rat myocardial infarction model through activation of endogenous regeneration. J. Control Release 173, 132-139.

Freeman, G.J., Casasnovas, J.M., Umetsu, D.T., and De Kruyff, R.H. (2010). TIM genes: A family of cell surface phosphatidylserine receptors that regulate innate and adaptive immunity. Immunol. Rev. 235, 172-189.

Gattazzo, F., Urciuolo, A., and Bonaldo, P. (2014). Extracellular matrix: A dynamic microenvironment for stem cell niche. Biochim. Biophys. Acta. 1840, 2506-2519.

Giordano, A., Galderisi, U., and Marino, I.R. (2007). From the laboratory bench to the patient's bedside: An update on clinical trials with mesenchymal stem cells. J. Cell Physiol. 211, 27-35.

Gnecchi, M., He, H., Noiseux, N., Liang, O.D., Zhang, L., Morello, F., Mu, H., Melo, L.G., Pratt, R.E., Ingwall, J.S., and Dzau, V.J. (2006). Evidence supporting paracrine hypothesis for Aktmodified mesenchymal stem cell-mediated cardiac protection and functional improvement. FASEB J. 20, 661-669.

Gu, X., Liu, X., Xu, D., Li, X., Yan, M., Qi, Y., Yan, W., Wang, W., Pan, J., Xu, Y., Xi, B., Cheng, L., Jia, J., Wang, K., Ge, J., and Zhou, M. (2010). Cardiac functional improvement in rats with myocardial infarction by up-regulating cardiac myosin light chain kinase with neuregulin. Cardiovasc. Res. 88, 334-343.

Gupta, D., Treon, S.P., Shima, Y., Hideshima, T., Podar, K., Tai, Y.T., Lin, B., Lentzsch, S., Davies, F.E., Chauhan, D., Schlossman, R.L., Richardson, P., Ralph, P., Wu, L., Payvandi, F., Muller, G., Stirling, D.I., and Anderson, K.C. (2001). Adherence of multiple myeloma cells to bone marrow stromal cells upregulates vascular endothelial growth factor secretion: Therapeutic applications. Leukemia 15, 1950-1961.

Halkein, J., Tabruyn, S.P., M. Ricke-Hoch, Haghikia, A., Nguyen, N.Q., Scherr, M., Castermans, K., Malvaux, L., Lambert, V., Thiry, M., Sliwa, K., Noel, A., Martial, J.A., Hilfiker-Kleiner, D., and Struman, I. (2013). MicroRNA-146a is a therapeutic target and biomarker for peripartum cardiomyopathy. J. Clin. Invest. 123, 2143-2154.

Hanahan, D., and Weinberg, R.A. (2011). Hallmarks of cancer: The next generation. Cell 144, 646-674.

Howcroft, T.K., Zhang, H.G., Dhodapkar, M., and Mohla, S. (2011). Vesicle transfer and cell fusion: Emerging concepts of cell-cell communication in the tumor microenvironment. Cancer Biol. Ther. 12, 159-164.
Hullin-Matsuda, F., Luquain-Costaz, C., Bouvier, J., and DeltonVandenbroucke, I. (2009). Bis(monoacylglycero)phosphate, a peculiar phospholipid to control the fate of cholesterol: Implications in pathology. Prostaglandins Leukot. Essent. Fatty Acids 81, 313-324.

Ikhapoh, I.A., Pelham, C.J., and Agrawal, D.K. (2015). Sry-type HMG box 18 contributes to the differentiation of bone marrow-derived mesenchymal stem cells to endothelial cells. Differentiation 89, 87-96.

Jelonek, K., Widlak, P., and Pietrowska, M. (2016). The influence of ionizing radiation on exosome composition, secretion and intercellular communication. Protein Pept. Lett. 23, 656663.

Johnstone, R.M. (2006). Exosomes biological significance: A concise review. Blood Cells Mol. Dis. 36, 315-321.

Katakowski, M., Buller, B., Zheng, X., Lu, Y., Rogers, T., Osobamiro, O., Shu, W., Jiang, F., and Chopp, M. (2013). Exosomes from marrow stromal cells expressing miR-146b inhibit glioma growth. Cancer Lett. 335, 201-204.

Katakowski, M., Zheng, X., Jiang, F., Rogers, T., Szalad, A., and Chopp, M. (2010). MiR-146b-5p suppresses EGFR expression and reduces in vitro migration and invasion of glioma. Cancer Invest. 28, 1024-1030.

Khan, M., Nickoloff, E., Abramova, T., Johnson, J., Verma, S.K., Krishnamurthy, P., Mackie, A.R., Vaughan, E., Garikipati, V.N., Benedict, C., Ramirez, V., Lambers, E., Ito, A., Gao, E., Misener, S., Luongo, T., Elrod, J., Qin, G., Houser, S.R., Koch, W.J., and Kishore, R. (2015). Embryonic stem cell-derived exosomes promote endogenous repair mechanisms and enhance cardiac function following myocardial infarction. Circ. Res. 117, 52-64.

Kumar, S., Witzig, T.E., Timm, M., Haug, J., Wellik, L., Kimlinger, T.K., Greipp, P.R., and Rajkumar, S.V. (2004). Bone marrow angiogenic ability and expression of angiogenic cytokines in myeloma: Evidence favoring loss of marrow angiogenesis inhibitory activity with disease progression. Blood 104, 1159-1165.

Lai, R.C., Arslan, F., Lee, M.M., Sze, N.S., Choo, A., Chen, T.S., M. Salto-Tellez, Timmers, L., Lee, C.N., El Oakley, R.M., Pasterkamp, G., de Kleijn, D.P., and Lim, S.K. (2010). Exosome secreted by MSC reduces myocardial ischemia/reperfusion injury. Stem Cell Res. 4, 214-222.

Lai, R.C., Chen, T.S., and Lim, S.K. (2011). Mesenchymal stem cell exosome: A novel stem cell-based therapy for cardiovascular disease. Regen. Med. 6, 481-492.

Lai, R.C., Tan, S.S., Teh, B.J., Sze, S.K., Arslan, F., de Kleijn, D.P., Choo, A., and Lim, S.K. (2012). Proteolytic potential of the MSC exosome proteome: Implications for an exosomemediated delivery of therapeutic proteasome. Int. J. Proteomics 2012, 971907.

Lee, J.K., Park, S.R., Jung, B.K., Jeon, Y.K., Lee, Y.S., Kim, M.K., Kim, Y.G., Jang, J.Y., and Kim, C.W. (2013). Exosomes derived from mesenchymal stem cells suppress angiogenesis by down-regulating VEGF expression in breast cancer cells. PLoS One 8, e84256.

Leiker, M., Suzuki, G., Iyer, V.S., Canty, J.M., Jr., and Lee, T. (2008). Assessment of a nuclear affinity labeling method for tracking implanted mesenchymal stem cells. Cell Transplant. 17, 911-922.

Llorente, A., Skotland, T., Sylvanne, T., Kauhanen, D., Rog, T., Orlowski, A., Vattulainen, I., Ekroos, K., and Sandvig, K. (2013). Molecular lipidomics of exosomes released by PC-3 prostate cancer cells. Biochim. Biophys. Acta. 1831, 13021309. 
Maegdefessel, L., Spin, J.M., Raaz, U., Eken, S.M., Toh, R., Azuma, J., Adam, M., Nakagami, F., Heymann, H.M., Chernogubova, E., Jin, H., Roy, J., Hultgren, R., Caidahl, K., Schrepfer, S., Hamsten, A., Eriksson, P., McConnell, M.V., Dalman, R.L., and Tsao, P.S. (2014). miR-24 limits aortic vascular inflammation and murine abdominal aneurysm development. Nat. Commun. 5, 5214.

Mathivanan, S., and Simpson, R.J. (2009). ExoCarta: A compendium of exosomal proteins and RNA. Proteomics 9, 49975000 .

Mirotsou, M., Jayawardena, T.M., Schmeckpeper, J., Gnecchi, M., and Dzau, V.J. (2011). Paracrine mechanisms of stem cell reparative and regenerative actions in the heart. J. Mol. Cell. Cardiol. 50, 280-289.

Miyanishi, M., Tada, K., Koike, M., Uchiyama, Y., Kitamura, T., and Nagata, S. (2007). Identification of Tim4 as a phosphatidylserine receptor. Nature 450, 435-439.

Mobius, W., van Donselaar, E., Ohno-Iwashita, Y., Shimada, Y., Heijnen, H.F., Slot, J.W., and Geuze, H.J. (2003). Recycling compartments and the internal vesicles of multivesicular bodies harbor most of the cholesterol found in the endocytic pathway. Traffic 4, 222-231.

Naito, Y., Yoshioka, Y., Yamamoto, Y., and Ochiya, T. (2017). How cancer cells dictate their microenvironment: Present roles of extracellular vesicles. Cell Mol. Life Sci. 74, 697713.

Nakamura, Y., Miyaki, S., Ishitobi, H., Matsuyama, S., Nakasa, T., Kamei, N., Akimoto, T., Higashi, Y., and Ochi, M. (2015). Mesenchymal-stem-cell-derived exosomes accelerate skeletal muscle regeneration. FEBS Lett. 589, 1257-1265.

Okazaki, T., Ebihara, S., Asada, M., Yamanda, S., Saijo, Y., Shiraishi, Y., Ebihara, T., Niu, K., Mei, H., Arai, H., and Yambe, T. (2007). Macrophage colony-stimulating factor improves cardiac function after ischemic injury by inducing vascular endothelial growth factor production and survival of cardiomyocytes. Am. J. Pathol. 171, 1093-1103.

Ono, M., Kosaka, N., Tominaga, N., Yoshioka, Y., Takeshita, F., Takahashi, R.U., Yoshida, M., Tsuda, H., Tamura, K., and Ochiya, T. (2014). Exosomes from bone marrow mesenchymal stem cells contain a microRNA that promotes dormancy in metastatic breast cancer cells. Sci. Signal 7, ra63.

Palmen, M., Daemen, M.J., De Windt, L.J., Willems, J., Dassen, W.R., Heeneman, S., Zimmermann, R., Van Bilsen, M., and Doevendans, P.A. (2004). Fibroblast growth factor-1 improves cardiac functional recovery and enhances cell survival after ischemia and reperfusion: A fibroblast growth factor receptor, protein kinase $\mathrm{C}$, and tyrosine kinase-dependent mechanism. J. Am. Coll. Cardiol. 44, 1113-1123.

Perrin-Cocon, L., Agaugue, S., Coutant, F., Masurel, A., Bezzine, S., Lambeau, G., Andre, P., and Lotteau, V. (2004). Secretory phospholipase A2 induces dendritic cell maturation. Eur. J. Immunol. 34, 2293-2302.

Qi, X., Zhang, J., Yuan, H., Xu, Z., Li, Q., Niu, X., Hu, B., Wang, Y., and Li, X. (2016). Exosomes secreted by humaninduced pluripotent stem cell-derived mesenchymal stem cells repair critical-sized bone defects through enhanced angiogenesis and osteogenesis in osteoporotic rats. Int. J. Biol. Sci. 12, 836-849.

Qian, L., van Laake, L.W., Huang, Y., Liu, S., Wendland, M.F., and Srivastava, D. (2011). miR-24 inhibits apoptosis and represses Bim in mouse cardiomyocytes. J. Exp. Med. 208, $549-560$

Qin, Y., Wang, L., Gao, Z., Chen, G., and Zhang, C. (2016). Bone marrow stromal/stem cell-derived extracellular vesicles regulate osteoblast activity and differentiation in vitro and promote bone regeneration in vivo. Sci. Rep. 6, 21961.

Quail, D.F., and Joyce, J.A. (2013). Microenvironmental regulation of tumor progression and metastasis. Nat. Med. 19, 1423-1437.

Radu, C.G., Yang, L.V., Riedinger, M., Au, M., and Witte, O.N. (2004). T cell chemotaxis to lysophosphatidylcholine through the G2A receptor. Proc. Natl. Acad. Sci. U. S. A. 101, 245250.

Record, M., Amara, S., Subra, C., Jiang, G., Prestwich, G.D., Ferrato, F., and Carriere, F. (2011a). Bis(monoacylglycero) phosphate interfacial properties and lipolysis by pancreatic lipase-related protein 2, an enzyme present in THP-1 human monocytes. Biochim. Biophys. Acta. 1811, 419-430.

Record, M., Carayon, K., Poirot, M., and Silvente-Poirot, S. (2014a). Exosomes as new vesicular lipid transporters involved in cell-cell communication and various pathophysiologies. Biochim. Biophys. Acta. 1841, 108-120.

Record, M., Poirot, M., and Silvente-Poirot, S. (2014b). Emerging concepts on the role of exosomes in lipid metabolic diseases. Biochimie 96, 67-74.

Record, M., Subra, C., Silvente-Poirot, S., and Poirot, M. (2011b). Exosomes as intercellular signalosomes and pharmacological effectors. Biochem. Pharmacol. 81, 1171-1182.

Roccaro, A.M., Sacco, A., Maiso, P., Azab, A.K., Tai, Y.T., Reagan, M., Azab, F., Flores, L.M., Campigotto, F., Weller, E., Anderson, K.C., Scadden, D.T., and Ghobrial, I.M. (2013). BM mesenchymal stromal cell-derived exosomes facilitate multiple myeloma progression. J. Clin. Invest. 123, 1542-1555.

Rombouts, K., Carloni, V., Mello, T., Omenetti, S., Galastri, S., Madiai, S., Galli, A., and Pinzani, M. (2013). Myristoylated Alanine-Rich protein Kinase C Substrate (MARCKS) expression modulates the metastatic phenotype in human and murine colon carcinoma in vitro and in vivo. Cancer Lett. 333, 244-252.

Sahu, R., Kaushik, S., Clement, C.C., Cannizzo, E.S., Scharf, B., Follenzi, A., Potolicchio, I., Nieves, E., Cuervo, A.M., and Santambrogio, L. (2011). Microautophagy of cytosolic proteins by late endosomes. Dev. Cell. 20, 131-139.

Salomon, C., Ryan, J., Sobrevia, L., Kobayashi, M., Ashman, K., Mitchell, M., and Rice, G.E. (2013). Exosomal signaling during hypoxia mediates microvascular endothelial cell migration and vasculogenesis. PLoS One 8, e68451.

Shao, L., Zhang, Y., Lan, B., Wang, J., Zhang, Z., Zhang, L., Xiao, P., Meng, Q., Geng, Y.J., Yu, X.Y., and Li, Y. (2017). MiRNA-sequence indicates that mesenchymal stem cells and exosomes have similar mechanism to enhance cardiac repair. Biomed. Res. Int. 2017, 4150705.

Singla, D.K. (2016). Stem cells and exosomes in cardiac repair. Curr. Opin. Pharmacol. 27, 19-23.

Steelman, L.S., Chappell, W.H., Abrams, S.L., Kempf, R.C., Long, J., Laidler, P., Mijatovic, S., Maksimovic-Ivanic, D., Stivala, F., Mazzarino, M.C., Donia, M., Fagone, P., Malaponte, G., Nicoletti, F., Libra, M., Milella, M., Tafuri, A., Bonati, A., Basecke, J., Cocco, L., Evangelisti, C., Martelli, A.M., Montalto, G., Cervello, M., and Mc Cubrey, J.A. (2011). Roles of the Raf/MEK/ERK and PI3K/PTEN/Akt/ mTOR pathways in controlling growth and sensitivity to therapy-implications for cancer and aging. Aging (Albany NY) 3, 192-222.

Strauss, K., Goebel, C., Runz, H., Mobius, W., Weiss, S., Feussner, I., Simons, M., and Schneider, A. (2010). Exosome secretion ameliorates lysosomal storage of cholesterol in 
Niemann-Pick type C disease. J. Biol. Chem. 285, 26279_ 26288.

Subra, C., Grand, D., Laulagnier, K., Stella, A., Lambeau, G., Paillasse, M., De Medina, P., Monsarrat, B., Perret, B., Silvente-Poirot, S., Poirot, M., and Record, M. (2010). Exosomes account for vesicle-mediated transcellular transport of activatable phospholipases and prostaglandins. J. Lipid Res. 51, 2105-2120.

Ulrich, H., do Nascimento, I.C., Bocsi, J., and Tarnok, A. (2015). Immunomodulation in stem cell differentiation into neurons and brain repair. Stem Cell Rev. 11, 474-486.

Valadi, H., Ekstrom, K., Bossios, A., Sjostrand, M., Lee, J.J., and Lotvall, J.O. (2007). Exosome-mediated transfer of mRNAs and microRNAs is a novel mechanism of genetic exchange between cells. Nat. Cell Biol. 9, 654-659.

Vallabhaneni, K.C., Penfornis, P., Dhule, S., Guillonneau, F., Adams, K.V., Mo, Y.Y., Xu, R., Liu, Y., Watabe, K., Vemuri, M.C., and Pochampally, R. (2015). Extracellular vesicles from bone marrow mesenchymal stem/stromal cells transport tumor regulatory microRNA, proteins, and metabolites. Oncotarget 6, 4953-4967.

van der Pol, E., Boing, A.N., Gool, E.L., and Nieuwland, R. (2016). Recent developments in the nomenclature, presence, isolation, detection and clinical impact of extracellular vesicles. J. Thromb. Haemost. 14, 48-56.

Wang, B., Komers, R., Carew, R., Winbanks, C.E., Xu, B., Herman-Edelstein, M., Koh, P., Thomas, M., Jandeleit-Dahm, K., Gregorevic, P., Cooper, M.E., and Kantharidis, P. (2012). Suppression of microRNA-29 expression by TGF-beta1 promotes collagen expression and renal fibrosis. J. Am. Soc. Nephrol. 23, 252-265.

Wang, K., Jiang, Z., Webster, K.A., Chen, J., Hu, H., Zhou, Y., Zhao, J., Wang, L., Wang, Y., Zhong, Z., Ni, C., Li, Q., Xiang, C., Zhang, L., Wu, R., Zhu, W., Yu, H., Hu, X., and Wang, J. (2017). Enhanced cardioprotection by human endometrium mesenchymal stem cells driven by exosomal microRNA-21. Stem Cells Transl. Med. 6, 209-222.

Wang, P., Zhang, Y., Li, H., Chieu, H.K., Munn, A.L., and Yang, H. (2005). AAA ATPases regulate membrane association of yeast oxysterol binding proteins and sterol metabolism. EMBO J. 24, 2989-2999.

Wang, X., Gu, H., Qin, D., Yang, L., Huang, W., Essandoh, K., Wang, Y., Caldwell, C.C., Peng, T., Zingarelli, B., and Fan, G.C. (2015). Exosomal miR-223 contributes to mesenchymal stem cell-elicited cardioprotection in polymicrobial sepsis. Sci. Rep. 5, 13721.

Wang, X., Huang, W., Liu, G., Cai, W., Millard, R.W., Wang, Y., Chang, J., Peng, T., and Fan, G.C. (2014). Cardiomyocytes mediate anti-angiogenesis in type 2 diabetic rats through the exosomal transfer of miR-320 into endothelial cells. J. Mol. Cell Cardiol. 74, 139-150.
Yang, W., Zheng, H., Wang, Y., Lian, F., Hu, Z., and Xue, S. (2015). Nesprin-1 has key roles in the process of mesenchymal stem cell differentiation into cardiomyocyte-like cells in vivo and in vitro. Mol. Med. Rep. 11, 133-142.

Yu, B., Gong, M., Wang, Y., Millard, R.W., Pasha, Z., Yang, Y., Ashraf, M., and Xu, M. (2013). Cardiomyocyte protection by GATA-4 gene engineered mesenchymal stem cells is partially mediated by translocation of miR-221 in microvesicles. PLoS One 8, e73304.

Yu, B., Kim, H.W., Gong, M., Wang, J., Millard, R.W., Wang, Y., Ashraf, M., and Xu, M. (2015). Exosomes secreted from GATA-4 overexpressing mesenchymal stem cells serve as a reservoir of anti-apoptotic microRNAs for cardioprotection. Int. J. Cardiol. 182, 349-360.

Zgheib, C., Zouein, F.A., Kurdi, M., and Booz, G.W. (2012). Chronic treatment of mice with leukemia inhibitory factor does not cause adverse cardiac remodeling but improves heart function. Eur. Cytokine Netw. 23, 191-197.

Zhang, H., Xiang, M., Meng, D., Sun, N., and Chen, S. (2016a). Inhibition of myocardial ischemia/reperfusion injury by exosomes secreted from mesenchymal stem cells. Stem Cells Int. 2016, 4328362.

Zhang, S., Chu, W.C., Lai, R.C., Lim, S.K., Hui, J.H., and Toh, W.S. (2016b). Exosomes derived from human embryonic mesenchymal stem cells promote osteochondral regeneration. Osteoarthritis Cartilage 24, 2135-2140.

Zhu, W., Huang, L., Li, Y., Zhang, X., Gu, J., Yan, Y., Xu, X., Wang, M., Qian, H., and Xu, W. (2012). Exosomes derived from human bone marrow mesenchymal stem cells promote tumor growth in vivo. Cancer Lett. 315, 28-37.

Address correspondence to:

Qing Gao

School of Integrative Medicine

Tianjin University of Traditional Chinese Medicine 88th Yuquan Road

Nankai District

Tianjin 300193

China

E-mail:616264342@qq.com

Xijuan Jiang

School of Integrative Medicine

Tianjin University of Traditional Chinese Medicine

88th Yuquan Road

Nankai District

Tianjin 300193

China

E-mail: xijuanjiang@foxmail.com 